\title{
Using Virtual Reality Tools for Teaching Foreign Languages
}

\author{
Bruno Peixoto $^{1(\otimes)}$, Darque Pinto ${ }^{1}$, Aliane Krassmann ${ }^{2}$, Miguel Melo $^{3}$, \\ Luciana Cabral ${ }^{4}$, and Maximino Bessa ${ }^{1,3}$ \\ 1 Universidade de Trás-os-Montes e Alto Douro, Vila Real, Portugal \\ brunomepeixoto@gmail.com \\ 2 Universidade Federal do Rio Grande do Sul, Porto Alegre, Brazil \\ 3 INESC TEC, Porto, Portugal \\ 4 CITCEM and Instituto Politécnico de Bragança, Bragança, Portugal
}

\begin{abstract}
Among the wide application areas that Virtual Reality (VR) can have a major impact, one is Education. However, this potential is still unexplored, and one of these gaps has to do with language learning. Listening activities, which are often only supported by audio, are thought of to be demanding area when it comes to learning a second or foreign language and so therefore an interesting area for VR to take place. This pilot study therefore presents the perceptions of foreign language teachers regarding a novel medium for delivering listening activities to their students: Virtual Reality technology. The results show that foreign language teachers are of the opinion that this technology can help motivate students and potentiate the student's learning curve regarding the listening of a foreign or second language.
\end{abstract}

Keywords: Virtual Reality · Media in education •

Teaching/learning strategies

\section{Introduction}

Nowadays in students' lives technology is everywhere, from interactive games to the growing plethora of digital devices. All this exposure to technology means that their learning expectations are likely to be very different from previous generations where technology was not widespread [1]. This scenario makes the traditional teaching techniques sometimes fail when it comes to engaging the contemporary students and, consecutively, creates an inability to motivate them to learn [2].

One of the most important factors regarding foreign or second language learning is to be exposed to it, by talking or listening to it. Listening sessions can be defined as when listeners are able to convert visual and auditory clues into information about what is going on in any given situation [3]. They are part of most language courses and international proficiency tests as the Test of English as a Foreign Language (TOEFL) TOEFL and the International English Language

(C) Springer Nature Switzerland AG 2019

Á. Rocha et al. (Eds.): WorldCIST'19 2019, AISC 932, pp. 581-588, 2019.

https://doi.org/10.1007/978-3-030-16187-3_56 
Testing System (IELTS), in the case of English language. In addition, they are the hardest ones, as it requires the student the ability to carefully pay attention to understand in real time what is being said.

The use of multimedia content in education is considered to be quite productive. Videos, images, sounds, animations or simulations are just a few types of multimedia that can be used in a meaningful way due to its interactivity and flexibility [4]. Virtual Reality (VR) technology, described as the use of an immersive computer-simulated environment in which people can interact with [5], incorporates these traditional multimedia and significantly elevates the level of user immersion, particularly at the level of visual perception. It replaces interaction with immersion; it replaces the desktop metaphor with a world metaphor; and it replaces direct manipulation with symbiosis [6]. The high levels of immersion, authenticity and interaction provided by VR allow the user to believe that he is within the computer-simulated environment [7], which is called the sense of presence. This aspect gives the opportunity to help students comprehend complex or abstract concepts in situations that have cost or security constrains or even which may no longer exist [8]. Studies by the VIRART group [9] from (PAÍS) concluded that VR applications are an asset in teaching, more specifically in especial communication languages.

From 2012 onwards, VR technology started to attract the public insterest with the success of Oculus Rift, a wearable and affordable Head Mounted Display (HMD), with stereoscopic displays that is considered to be comfortable and lightweighted. In 2014 Google launched Google Cardboard, which could turn any smartphone in an HMD. Due to this recent expansion and cost reduction, few studies have already investigated the very benefits of VR in foreign or second language teaching and learning, being mostly restricted to theoretical ones. Schwienhorst [10], for example, reports that the investigation of VR field in foreign language teaching is still largely unexplored, although incredibly helpful not only to bring language learners closer to the language culture but also to create realistic simulations that wouldn't even exist in the real world. Lin and Lan [11], by their turn, suggest that foreign language teachers are now expected to employ VR technologies as a way to help their students, which changes the teacher's role from the person with all the answers to facilitators who support students in the virtual world path [9]. In contrast, most of today's teachers still lack the knowledge and skills regarding this use, making them reluctant to incorporate new media into their teaching $[12,13]$. Thus, according to Youngblut [9], VR developers and teachers should work together to overcome this impasse.

Bearing in mind the above, we developed an immersive VR experience aimed at English language teaching, focusing in the listening activities. In order to verify if teachers can see the benefits of VR to improve the classic listening exercises or not, we conducted a pilot study having a teacher-based sample to investigate the experience. The research was therefore carried out together in a private school, the English Institute of Vila Real, Portugal, with seven foreign language teachers familiarised with the traditional listening exercises and for 
whom the VR technology and educational tools were yet to be experimented and applied to language teaching and learning.

\section{Methods}

Listening comprehension, especially in foreign language learners, requires the listener to observe attentively as much visual and auditory clues as possible inserted in an audio, in order to have a real idea of what is going on in the given narrative. Since in most basic and language schools the listening exercises are mostly supported by audio streaming, an immersive VR application was developed to help teachers explain a particular scenario in a listening session. Four from the seven teachers who participated in the pilot study were not only responsible for the dialogue script for the VR application but also gave voice to Non-Player Characters (NPCs). The methodology adopted consists of a quasi-experimental design, cross-sectional study with a quantitative focus. The sampling technique used was the nonprobabilistic convenience sampling procedure.

\subsection{Sample}

The sample consisted of teachers. Namely, seven participants (six female and one male), between 35 and 53 years old $(M=44,50, S D=6,02)$ who were foreign language teachers from the English Institute of Vila Real, in Portugal. The teachers were accustomed to deal with listening exercises by means of audio in their classes and had no previous VR experience.

\subsection{Materials}

A laptop computer was used for the experiments. It had Intel Core i7-7700HQ processor and NVIDIA GeForce 1070 graphics card; it was responsible for running the game engine and all the input and output devices required for the experiment were connected to it. For the visual stimulus, the HTC Vive HMD was used. This $\mathrm{HMD}$ features a $110^{\circ}$ viewing angle and a resolution of $1080 \times 1200$ pixels per eye. The audio stimulus was delivered with a noise cancellation headphones (Bose QuietComfort 25).

In order to support the study, a realistic virtual environment (VE) was created by using the Unity game engine and it consist of two scenarios (Fig. 1, one representing a formal language dialogue, consisting of an office, and the other an informal one, consisting of an English pub.

The scenarios were filled with props that characterize an office room and a pub, respectively, and NPCs to meet the dialogue script. In both scenarios, the user would be placed at a table surrounded by the NPCs, as if he/she is part of the plot. The movement of the characters was produced with motion capture animation to ensure a realistic simulation, using an OptiTrack tracking system with 8 Prime 13 cameras. 


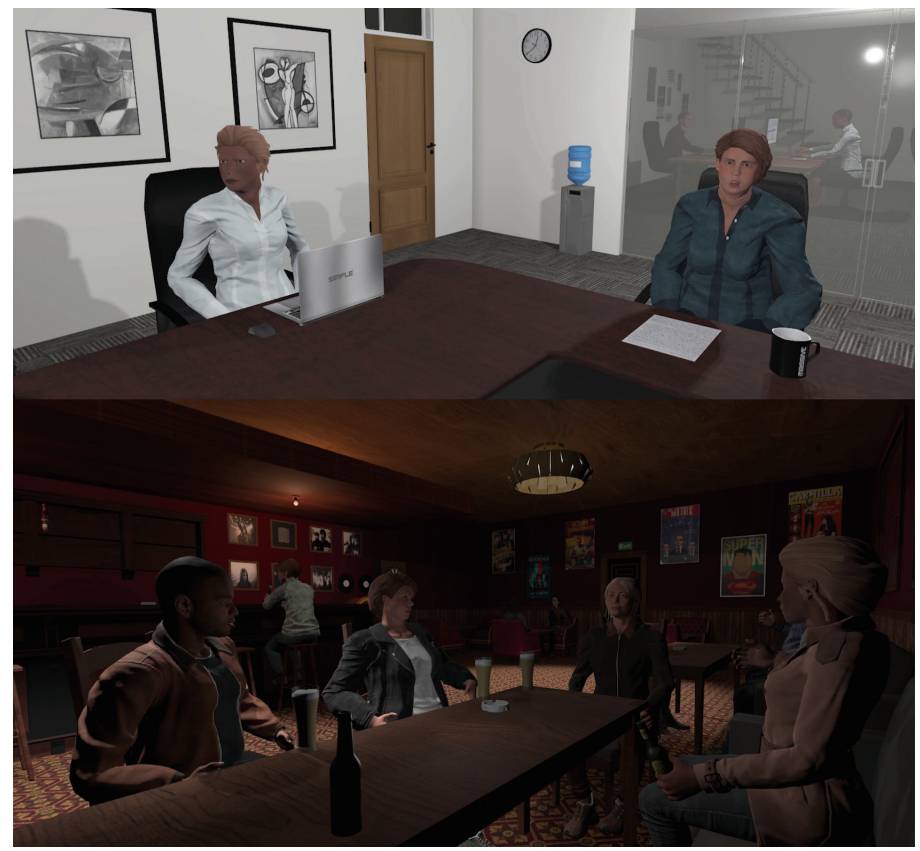

Fig. 1. View of the VE during the formal scenario (top) and informal scenario (bottom)

Each virtual scenario was developed having into account the study by Melo et al. [14] to ensure that Presence was not affected by the exposure time. Thus, the formal scenario had a length of 2:36 minutes and the informal scenario a length of $1: 29 \mathrm{~min}$.

\subsection{Instruments}

Concerning the questionnaires as presented: (1) a simple sociodemographic questionnaire to determine the sample characteristics and teaching experiences regarding multimedia content in class; (2) a 6 item 7-point Likert scale questionnaire based in After-Scenario Questionnaire (ASQ) to assess the teachers' satisfaction with the virtual experience [15]. (3) a 4 item 7-point Likert scale created by the authors to understand the teachers' satisfaction with the immersive VR application as an educational tool. The questionnaires were presented in printed format in the teachers' first language, Portuguese. A rough translation of the questionaries (2) and (3) is presented below.

(2) Questionnaire based in After-Scenario Questionnaire (ASQ):

In general...

... I liked the experience I just had

... The experiment discouraged me 
... I felt discomfort throughout the experience

... I'm satisfied with the ease with which the tasks were completed

... I'm satisfied with the time it took to complete the tasks

... I'm satisfied with the information given by the application.

(3) Questionnaire regarding the immersive VR application as an educational tool:

In general...

... I am satisfied with this application as a teaching method

... I believe this experiment complements in a positive way the traditional listening exercise

...I believe this experiment facilitates teaching techniques

...I would use this technology as a method of teaching in my classes.

\subsection{Procedure}

All experiments were conducted in a classroom at the English Institute of Vila Real, where external variables were controlled. Each teacher participated individually. It started with instructions about the experiment and with the participants filling in the sociodemographic questionnaire in the sequence. Afterwards, they were asked to seat, and the HMD and the headphones were properly placed with the help of the researchers.

The experiment was preceded by a pre-exposure in the VE, where the participants were given a period of around one minute to get acquainted with the VE. In this phase, the participant was immersed in the respective scenario, but without the NPCs nor the dialogue. This phase happened before each scenario. After that period, the NPCs appeared and the dialogue started right away.

After ending the two VR scenarios, the participants got help with the removal of all the equipment and were guided to a table so that they could complete the remaining questionnaires.

Overall, every participant took between 15 to $20 \mathrm{~min}$ to finish the whole procedures, including the questionnaires.

\section{Results}

The sociodemographic data shows that $85,7 \%$ of the participants use multimedia content in class at least occasionally $(M=1,14, S D=0,53)$. When inquired the types of multimedia they use, $100 \%$ answered sounds (music, recordings of voice, etc.), $85,7 \%$ images (photographs, drawings, maps, figures, etc.), $57,1 \%$ videos (entertainment, educational, animations, etc.), and only $14,3 \%$ reported the use of simulations and others. It was questioned what was their perceived level of knowledge on VR, in which $71,4 \%$ of the participants said that they only have basic knowledge about this technology and how it works.

The scores for user satisfaction regarding the virtual experience (ASQ) were very positive with an overall mean of 6,81 and a standard deviation of 0,78 in 


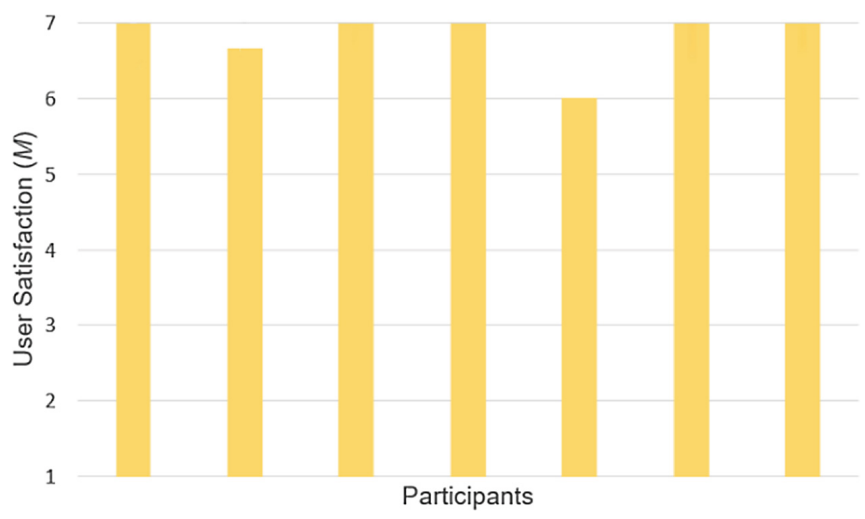

Fig. 2. User satisfaction regarding the virtual experience

a scale from 1 to 7 , where a higher value represents a higher user satisfaction (Fig. 2).

Regarding to the teachers' satisfaction with the immersive VR application as an educational tool, the results were even more positive, with $\mathrm{M}=6,86$ and $\mathrm{SD}=0,45$ (Fig. 3). A closer analysis revealed that the participants strongly agree that: (1) this experience complements in a positive way the classic listening exercise; (2) this experiment facilitates and helps with the language teaching methods; (3) they would like to use this technology as a way of teaching in their classes.

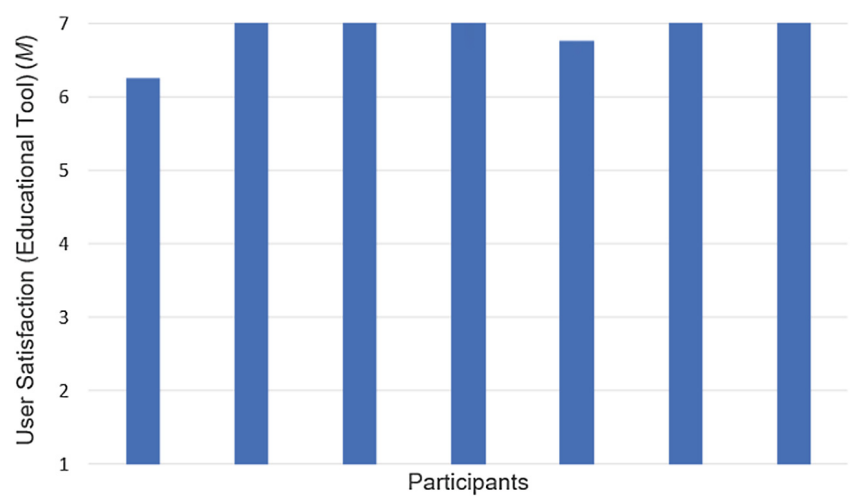

Fig. 3. User satisfaction regarding the VR application as an educational tool

\section{Discussion}

Since its emergence, digitally generation has been losing interest in the traditional teaching techniques $[1,2]$. So nowadays, the teacher's role is to try and 
convert the established learning theories into new practices [1] while guiding, supporting and facilitating their students' learning [4]. Since foreign language learners should convert both visual and auditory clues into information [3] and most schools only use audio in listening exercises, an immersive VR application regarding this topic seems ideal to help the student, while motivating them by providing a new way of learning.

Rickel and Johnson [16] point out that VR makes simulations closer to reallife experiences, because instead of observing the simulated world through a workspace window, students are immersed in a 3D environment where they can improve their skills through more realistic practices. Thus, VR adds another layer of interactivity which could substantially improve the students' concentration during listening exercises, and as a consequence, help them to understand an memorize what is being said. In addition, it allows to place the student in a foreign experience, in a simulated environment that resembles and evokes very culture, society and country of the language being learned, that is, the real context of the language.

Even though teachers seem to be hesitant to incorporate new digital media into their teaching [2], our results show that they are willing to use VR as a teaching technique and take advantage of the mentioned benefits. However, since the students will be unaware of their surroundings while using HMD, constant supervision is needed to assure their safety, especially with the minors. As the results also show, teachers are unfamiliar with this technology, so convincing them of its benefits is the first step towards this incorporation, in which this study made a contribution.

\section{Conclusion}

The main objective of this pilot study was to evaluate the perceptions of foreign language teachers regarding the benefits of a VR application as a tool to help their students with listening sessions, rather than the classic listening exercises where the student only has access to audio. To this end, a VR application was developed which allowed the user to be immersed in the virtual world representing formal and informal scenarios. After testing it with foreign language teachers, we concluded that there is a unanimous opinion that such technology is not only attractive but can also help to motivate students and potentiate the student's learning curve regarding the listening of a foreign language.

The main limitation of the pilot study is the sample size. Future work intends to broaden the scope of the study to both get, a larger sample, with more teachers and schools participating and, extend this VR-based learning tool to incorporate more learning activities. Afterwards, with the lessons learned we plan to design a pedagogic framework that could help teachers in making successful use of VR, towards more digitally enhanced classes. 
Acknowledgments. This work is financed by the ERDF - European Regional Development Fund through the Operational Programme for Competitiveness and Internationalisation - COMPETE 2020 Programme and by National Funds through the Portuguese funding agency, FCT - Fundação para a Ciência e a Tecnologia within project POCI-01-0145-FEDER-028618 entitled PERFECT - Perceptual Equivalence in virtual Reality For authEntiC Training.

We would like to thank Claúdia Peixoto, Marta Peixoto and the other foreign languages teachers from the English Institute of Vila Real for their collaboration in this experience.

\section{References}

1. Wang, R., Newton, S., Lowe, R.: Experiential learning styles in the age of a virtual surrogate. Int. J. Arch. Res. ArchNet-IJAR 9, 93-110 (2015). https://doi.org/10. 26687/archnet-ijar.v9i3.715

2. Prensky, M.: Digital Game-Based Learning. Paragon House, New York (2007)

3. Alatis, J.: Georgetown University Round Table on Languages and Linguistics (GURT) 1990: Linguistics, Language Teaching and Language Acquisition: The Interdependece of Theory, Practice and Research, Georgetown University Round Table on Languages and Linguistics series. Georgetown University Press (1990)

4. Andresen, B., van den Brink, K., Unesco Institute for Information Technologies in Education: Multimedia in Education: Curriculum. Unesco Institute for Information Technologies in Education (2013)

5. Machover, C., Tice, S.E.: Virtual reality. IEEE Comput. Graph. Appl. 14(1), 15-16 (1994). https://doi.org/10.1109/38.250913

6. Psotka, J.: Immersive training systems: virtual reality and education and training. Instr. Sci. 23(5), 405-431 (1995). https://doi.org/10.1007/BF00896880

7. Alqahtani, A.S., Daghestani, L.F., Ibrahim, L.F.: Environments and system types of virtual reality technology in stem: a survey. Int. J. Adv. Comput. Sci. Appl. 8(6). https://doi.org/10.14569/IJACSA.2017.080610

8. Christou, C.: Virtual Reality in Education, Ch. 12, pp. 228-243. IGI Global (2010). https://doi.org/10.4018/978-1-60566-940-3.ch012

9. Youngblut, C.: Educational Uses of Virtual Reality Technology. Institute for Defense Dnalyses (1998)

10. Schwienhorst, K.: The state of VR: a meta-analysis of virtual reality tools in second language acquisition. Comput. Assist. Lang. Learn. 15(3), 221-239 (2002). https:// doi.org/10.1076/call.15.3.221.8186

11. Lin, T.-J., Lan, K.: Language learning in virtual reality environments: past, present, and future. Educ. Technol. Soc. 18, 486-497 (2015)

12. Prensky, M.: Don't Bother Me Mom-I'm Learning!, 1st edn. Paragon House, New York (2006)

13. Albion, P.: Self-efficacy beliefs as an indicator of teachers' preparedness for teaching with technology. Creative Education

14. Melo, M., Vasconcelos-Raposo, J., Bessa, M.: Presence and cybersickness in immersive content: effects of content type, exposure time and gender. Comput. Graph. 71, 159-165 (2018)

15. Lewis, J.R.: IBM computer usability satisfaction questionnaires: psychometric evaluation and instructions for use. Int. J. Hum. Comput. Interact. 7(1), 57-78 (1995). https://doi.org/10.1080/10447319509526110

16. Johnson, W., Rickel, J.: Steve: an animated pedagogical agent for procedural training in virtual environments. SIGART Bull. 8, 16-21 (1997) 